\title{
The Optimal Graph Whose Least Eigenvalue is Minimal among All Graphs via 1-2 Adjacency Matrix
}

\author{
Lubna Gul, ${ }^{1}$ Gohar Ali $\mathbb{D},{ }^{1}$ Usama Waheed, ${ }^{1}$ and Nudrat Aamir $\mathbb{D}^{2}$ \\ ${ }^{1}$ Department of Mathematics, Islamia College Peshawar, Peshawar, Pakistan \\ ${ }^{2}$ Department of Basic Science and Humanities, CECOS University of IT \& Emerging Sciences, Peshawar, Pakistan
}

Correspondence should be addressed to Gohar Ali; gohar.ali@icp.edu.pk and Nudrat Aamir; nudrat@cecos.edu.pk

Received 28 September 2021; Accepted 27 October 2021; Published 17 November 2021

Academic Editor: Elena Guardo

Copyright (c) 2021 Lubna Gul et al. This is an open access article distributed under the Creative Commons Attribution License, which permits unrestricted use, distribution, and reproduction in any medium, provided the original work is properly cited.

All graphs under consideration are finite, simple, connected, and undirected. Adjacency matrix of a graph $G$ is 0,1 matrix $A=\left[a_{i j}\right]=\left\{\begin{array}{l}0, \text { if } v_{i}=v_{j} \text { or } d\left(v_{i}, v_{j}\right) \geq 2 . \text {. Here in this paper, we discussed new type of adjacency matrix known by } 1-2 \text { adjacency } \\ 1, \text { if } d\left(v_{i}, v_{j}\right)=1 .\end{array}\right.$ matrix defined as $A_{1,2}(G)=\left[a_{i j}\right]=\left\{\begin{array}{l}0, \text { if } v_{i}=v_{j} \text { or } d\left(v_{i}, v_{j}\right) \geq 3 \\ 1, \text { if } d\left(v_{i}, v_{j}\right)=2\end{array}\right.$, from eigenvalues of the graph, we mean eigenvalues of the 1-2 adjacency matrix. Let $T_{n}^{c}$ be the set of the complement of trees of order $n$. In this paper, we characterized a unique graph whose least eigenvalue is minimal among all the graphs in $T_{n}^{c}$.

\section{Introduction}

Let $G=(V, E)$ be a simple graph with vertex set $V=V(G)$ and edge set $E=E(G)$. Its order is $|V(G)|$, denoted by $n$, and its size is $|E(G)|$, denoted by $m$. The distance between two vertices $u$ and $v$ of a graph $G$ is the length of a shortest path between them. The 1-2 adjacency matrix of $G$ is defined to be the matrix $A_{1,2}(G)=\left[a_{i j}\right]$ of order $n$ with $a_{i j}=1$ if $1 \leq d\left(v_{i}, v_{j}\right) \leq 2$ and $a_{i j}$ is zero for the rest cases. The solution of $\operatorname{det}\left(x I-A_{1,2}(G)\right)$ is the eigenvalues of $G$. Since $A_{1,2}(G)$ is always symmetric and real, all the eigenvalues can be arranged as $\lambda_{1}(G) \leq \lambda_{2}(G) \leq \ldots \leq \lambda_{n}(G)$. We write $\lambda_{\min }(G)$ for the least eigenvalue of $G$. One can find that $\lambda_{n}(G)$ is exactly the spectral radius of $G$.

The least eigenvalue of any graph is nonpositive. For only disconnected graph, it is equal to zero. Otherwise, for graphs with at least one edge, it is less than or equal to -1 (by the interlacing theorem, see [1], p-19); it is equal to -1 if and only if $G$ is a disjoint union of complete graphs or equivalently $G^{c}$ is a null graph or a complete multipartite graph. If $G$ contains $K_{1,2}$ as an induced subgraph, then $\lambda_{\text {min }}(G) \leq \lambda_{\text {min }}\left(K_{1,2}\right)=-\sqrt{2}$ (again by the interlacing theorem). For $1-2$ adjacency matrix, $\lambda_{\min }\left(K_{1,2}\right)=-1$, and if a graph $G$ containing $P_{5}$ is an induced subgraph, then $\lambda_{\min }(G) \leq 1$.

There are many results in the literature concerning the largest eigenvalue (spectral radius) of simple graph; see, e.g., [2] or [1]. Javaid examined different families of graphs to pick optimal graph with respect to least eigenvalues via usual adjacency matrix in their respective graph classes in [3-5]. Lubna et al. examined square power graph of $G$ for their least eigenvalue [6]. Recently, the problem of minimizing the least eigenvalue of graphs subject to one or more parameters has received more attention. Bell et al. [7, 8] discussed the least eigenvalue of connected graphs with prescribed order and size. Fan et al. [9] discussed the least eigenvalue of complement of trees. Liu et al. [10] discussed the least eigenvalue of unicyclic graphs with pendant vertices. Fan et al. [9] discussed the least eigenvalue of unicyclic graphs with given girth. Petrovic et al. [11] discussed the least eigenvalue of bicyclic graphs.

In a family of graphs, a graph is called minimizing if the least eigenvalue of its adjacency matrix is minimum in the set of the least eigenvalues of all the graphs. Denote by $T_{n}, T_{n}^{c}$ the set of trees of order $n$ and the set of the complements of trees order $n$, respectively. In this paper, we determine the 
unique minimizing graph in $T_{n}^{c}$ for $n \geq 6$, which is not the complement of the star graph, and we are limiting our discussion to the set $T_{n}^{c}-K_{n}^{c}$ where $K_{n}{ }^{c}$ denotes all those graphs whose $\lambda_{\min }$ is -1 .

Theorem 1 (see [7]). If $G$ is a graph whose least eigenvalue is minimal among all the connected graphs, then

(i) $G$ is either a bipartite graph

(ii) $G$ is a join of two nested split graphs

Theorem 2 (see [12]). Let $K(n, m)$ denote the family of all connected bipartite graphs and let $G \in K(n, m)$ be the graph such that its least eigenvalue is minimal among all the connected bipartite graphs, then $G$ must be a double nested graph.

\section{Main Results}

We begin with some definitions. Given a graph $G$ of order $n$, a vector $X \in R^{n}$ is called defined on $G$ if there exists an injective function $f$ from $V(G)$ to the entries of $X$; simply written $X_{v}=f(v)$ for each $v \in V(G)$, if $X$ is an eigenvector of $A_{1,2}(G)$, then it is naturally defined on $V(G)$ and every entry of $X$ corresponds to the vertex $v \in V(G)$. One can easily prove that

$$
X^{T} A_{1,2}(G) X=2 \sum_{d(u, v) \in\{1,2\}} X_{u} X_{v},
$$

and $\lambda$ is an eigenvalue of $G$ corresponding to the eigenvector $X$ iff $X \neq 0$ and

$$
\lambda X_{u}=\sum_{v \in N_{G}(u)} X_{v}, \quad \text { for each vertex } u \in V(G),
$$

where $N_{G}(u)$ denotes the neighbourhood of $u$ in the graph $G$. Equation (2) is called the eigen equation for the graph $G$. Now, for any arbitrary unit vector $X \in R^{n}$,

$$
\lambda_{\text {min }}(G) \leq X^{T} A_{1,2}(G) X,
$$

and it will be equal if and only if $X$ is a least eigenvector of $G$. Now, let $G^{c}$ denote the complement of a graph $G$. One can easily proof that $A_{1,2}\left(G^{c}\right)=J-I-A_{1,2}(G)$, where $I$ and $J$ denote the identity matrix and ones matrix of the same size as $A_{1,2}(G)$. Now, for any vector $X$,

$$
X^{T} A_{1,2}\left(G^{C}\right) X=X^{T}(J-I) X-X^{T} A_{1,2}(G) X .
$$

A graph $G$ is said to be a tree if and only if there is a path between any pair of vertices and has no cycle. A tree is said to be a star if and only if there exists a vertex of degree $n-1$ and all other vertices have degree 1 . We introduce a special tree, denoted by $T(p, q)$, which is obtained from two disjoint stars $K_{1, p}(p \geq 1)$ and $K_{1, q}(q \geq 1)$ by joining one pendant vertex of $K_{1, p}$ and one pendant vertex of $K_{1, q}$. The complement of $T(p, q)$ is $G^{c}=(T(p, q))^{c}$, as shown in Figure 1 .

Lemma 1. Given $n \geq 6$, for any positive integer $p, q$ such that $p \geq q \geq 1 \quad$ and $\quad p+q+2=n, \quad \lambda_{\min }(T(p, q))^{c}\{1,2\} \geq$

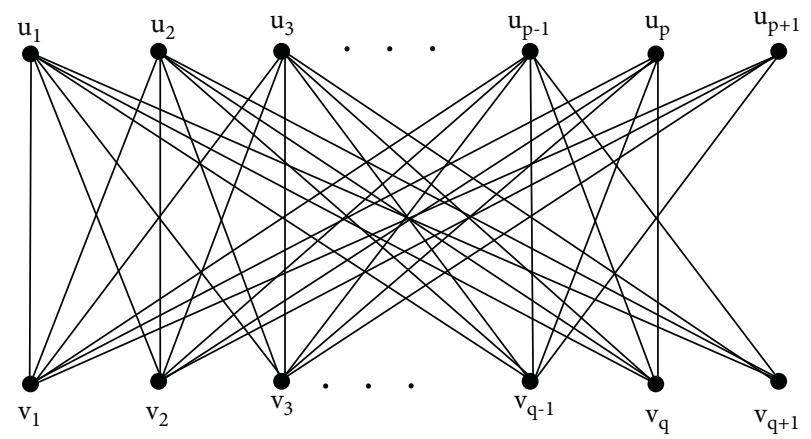

Figure 1: $G^{c}=(T(p, q))^{c}$ graph with $m=p q+p+q-2$.

$\lambda \min \left(T([(n-2) / 2],[(n-2) / 2])^{c}\right)\{1,2\}$, with equality if and only if $p=[(n-2) / 2]$ and $q=[(n-2) / 2]$.

Proof. Suppose $n \geq 6$. Let $\lambda_{1}$ be the least eigenvalue of $(T(p, q))^{c}$ and $X$ be the corresponding least eigenvector. Let $X_{i}$ denote the entries of $X$ corresponding to the vertex $v_{i}$ for $i=1,2,3,4,5,6$. Now, by eigen equation (2), all the vertices attached at $v_{2}$ and $v_{5}$ have the same values given by $X$, say $X_{1}$ and $X_{6}$. Now, as $\lambda_{\min }(T(p, q))^{c}<-1$, so by an eigen equation,

$$
\begin{aligned}
& \lambda_{1} X_{1}=X_{4}+X_{5}+(q-1) X_{6}, \\
& \lambda_{1} X_{2}=X_{5}+(q-1) X_{6}, \\
& \lambda_{1} X_{3}=(q-1) X_{6}, \\
& \lambda_{1} X_{4}=(p-1) X_{1}, \\
& \lambda_{1} X_{5}=(p-1) X_{1}+X_{2}, \\
& \lambda_{1} X_{6}=(p-1) X_{1}+X_{2}+X_{3} .
\end{aligned}
$$

Transform (5) into a matrix equation $\left(B-\lambda_{1} I\right) X^{\prime}=0$, where $X^{\prime}=\left(X_{1}, X_{2}, X_{3}, X_{4}, X_{5}, X_{6}\right)^{T}$ and the matric $B$ of order 6 is omitted. We get

$$
\begin{aligned}
f(\lambda ; p, q) & =\operatorname{det}(B-\lambda I)=(p+q-p q-1) \\
& -\lambda^{2}(2 p+2 q-3 p q-1)-\lambda^{4}(p+q+p q-2)+\lambda^{6},
\end{aligned}
$$

and

$f(\lambda ; p, q)-f(\lambda ; p-1, q+1)=(p-q-1)\left(\lambda^{4}-3 \lambda^{2}+1\right)$.

Note that $\lambda_{1}$ is a least root of $f(\lambda ; p, q)$. In addition, $f(-1,6180 ; p, q)=33.2670+0.00024593(p q)-$

$11.0894(p+q)<0$ as $p+q=n-2 \geq 4$, which implies $\lambda_{1}<-1.6180$.

If $p \geq q+2$, by the above discussion, for $\lambda_{1}<-1.6180$, we have

$$
f(\lambda ; p, q)-f(\lambda ; p-1, q+1)<0 .
$$

In particular, $f\left(\lambda_{1} ; p-1, q+1\right)<0$, which implies

$$
\lambda_{\text {min }}\left(T(p, q)^{c}\right) \geq \lambda_{\text {min }}(T(p-1, q+1))^{c} .
$$

The result now follows. 
Lemma 2. Let $T^{c}$ be a graph in $T_{n}{ }^{c}-K_{n}{ }^{c}$ and let $X$ be the least eigenvector of $T$, then $X$ contains at least two positive and two negative entries.

Proof. Assume on contrary that $X$ contains exactly one positive entry corresponding to the vertex $w$, i.e., $X_{w}>0$. Since $T$ is not a star, there exists a vertex $u \in N_{T}(w)$ such that $N_{T}(u) \backslash w \neq 0$. Considering the eigen equation (2) on the vertex $u$ for the graph $T^{c}$, we have

$$
0 \leq \lambda_{1}\left(T^{c}\right) X_{u}=\sum_{d(v, u) \in\{1,2\}} X_{v} \leq 0 .
$$

This implies that $X_{u}=0$ and $X_{v}=0$ for each $v \in N_{T}^{c}(u)$, now as

$$
\lambda_{1}\left(T^{c}\right) X_{w}=\sum_{d(v, w) \in\{1,2\}} X_{v} .
$$

Now, let $v_{0} \in N_{T}(u), w$. Then, by (2) and (11),

$$
\begin{aligned}
{\left[1+\lambda_{1}\left(T^{c}\right)\right] X_{v_{0}} } & =X_{v_{0}}+\sum_{v \in d\left(v, v_{0}\right) \in\{1,2\} \backslash v_{0}} X_{v}, \\
& =\sum_{v \in d\left(v, v_{0}\right) \in\{1,2\}} X_{v}, \\
& =X_{w}+\sum_{d\left(v, v_{0}\right) \in\{1,2\} \backslash w} X_{v}, \\
& =X_{w}+\lambda_{1}\left(T^{c}\right) X_{w}, \\
& =\left[1+\lambda_{1}\left(T^{c}\right)\right] X_{w} .
\end{aligned}
$$

This implies $X_{v_{0}}=X_{w}$. Since $\lambda_{1}\left(T^{c}\right)<1$ as $T$ is not a star, a contradiction, so our supposition is wrong and hence $X$ contains at least two positive entries. Similarly, if we consider $-X$, we also get $X$ which contains at least two negative entries. The result follows.

Before we state other results, we need some definitions.

Let $G$ be a bipartite graph with colour classes $U$ and $V$ such that $V(G)=U \cup V$. $G$ is said to be a double nested graph if $U=\left\{U_{1} \cup U_{2} \cup \ldots \cup U_{k}\right\} \quad$ and $V=\left\{V_{1} \cup V_{2} \cup \ldots \cup V_{k}\right\}$ such that each $u_{i} \in U_{1}$ is adjacent to each and every vertex in $V$, and similarly, each $u_{i} \in U_{2}$ is adjacent to each and every vertex in $V=\left\{V_{1} \cup V_{2} \cup \ldots \cup V_{k-1}\right\}$ for $i=\{1,2,3, \ldots, k\}$.

Now, as we know from [12], if the two vertices $u$ and $w$ belong to the same colour class and $x_{u} \geq x_{w}$, then $\operatorname{deg}(u) \geq \operatorname{deg}(w)$.

Let $U=\left\{u_{1}, u_{2}, \ldots, u_{k}\right\}$ and $V=\left\{v_{1}, v_{2}, \ldots, v_{k}\right\}$ be the colour classes such that $x_{u_{1}} \geq x_{u_{2}} \geq x_{u_{3}} \geq \ldots x_{u_{k}} \geq$ and $x_{v_{1}} \geq x_{v_{2}} \geq x_{v_{3}} \geq \ldots x_{v_{k}} \geq$, where $x_{u_{i}}$ and $x_{v_{i}}$ for $i=\{1,2,3, \ldots, k\}$ are the entries of the corresponding eigenvector $X$ of $G$.

Lemma 3 (see [12]). Let $G$ be a graph with the above assumptions. Then,

(1) The vertices $u_{1}$ and $v_{1}$ are adjacent

(2) The degree of $u_{1}$ and $v_{1}$ is complete

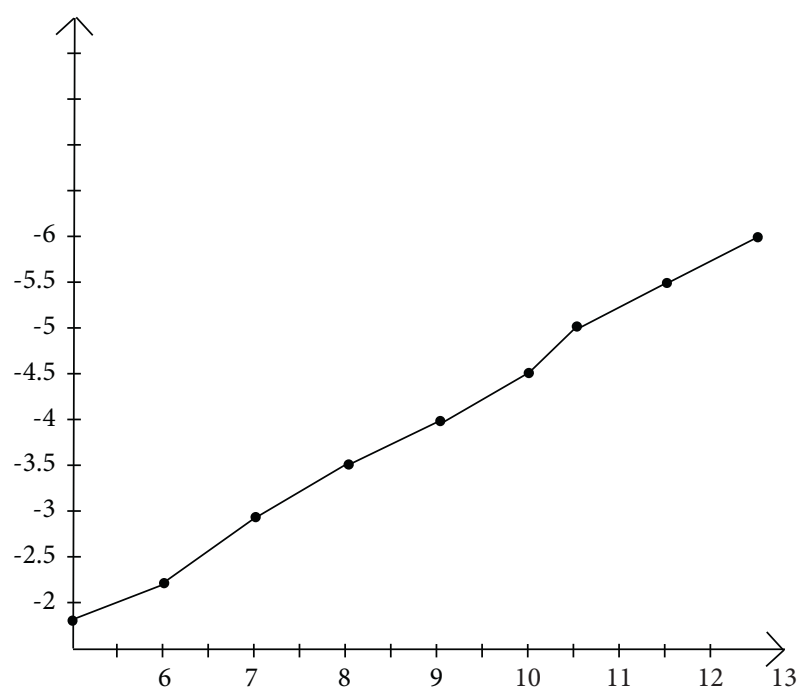

FIgURE 2: The behavior of eigenvalues with respect to number of vertices.

(3) If the vertex $u$ is adjacent to $v_{i}$, then $u$ is adjacent to $v_{j}$ for $j<k$, and if the vertex $v$ is adjacent to $u_{i}$, then $v$ is adjacent to $u_{j}$ for $j<k$

Observation 1. If $G$ is a bipartite of order $n$ and size $m$, then $\lambda_{\min } G$ goes to its lower bound by increasing the size of $G$.

Bell [12] discussed the behavior of maximum eigenvalue by increasing the number of edges and kept $V(G)$ fixed. We here show the behavior of least eigenvalue of a $(T(p, q))^{c}$ graph by fixing the number of edges at $m=p q+p+q-2$ and let the number of vertices be increased. In Figure 2, the horizontal axes show vertices and vertical axes represent least eigenvalues.

Theorem 3. Let $T$ be a tree of order $n \geq 4$. Then,

$$
\lambda_{\min }\left(T^{c}\right)_{\{1,2\}} \geq \lambda_{\min }\left(T\left(\frac{(n-2)}{2}, \frac{(n-2)}{2}\right)^{c}\right)_{\{1,2\}} .
$$

Proof. As we know,

$$
\lambda_{\min }(T(p, q))_{\{1,2\}}^{c} \geq \lambda_{\min }\left(T\left(\left[\frac{(n-2)}{2}\right],\left[\frac{(n-2)}{2}\right]\right)^{c}\right)_{\{1,2\}} .
$$

By Theorems 1 and 2, it is enough to show that $\left(T(p, q)^{c}\right)$ is a largest double nested graph at $1-2$ distance. Let $\left(T(p, q)^{c}\right)$ be the graph such that $\lambda_{\min }\left(T(p, q)^{c}\right)$ is the least one among all the graphs and let $X$ be the least eigenvector. Now, let $G$ be the graph obtained from $\left(T(p, q)^{c}\right)$ by rotating the edge $r_{i} s$ to $r_{i} t$ such that $s \ngtr t$ for $i=\{1,2, \ldots, k\}$, then $\lambda_{\min } G \leq \lambda_{\min }\left(T(p, q)^{c}\right)$, and similarly, if $s$ and $t$ belong to the same colour class s.t $x_{s} \geq x_{t}$, then $\operatorname{deg}(\mathrm{s}) \geq \operatorname{deg}(\mathrm{t})$, and so by Lemma 3 , it will be a double nested graph. Next, we will show that it is the largest possible double nested graph at $1-2$ distance. As the size $\left(T(p, q)^{c}\right)$ is $p q-3$, so all we need to show is that the size cannot exceed 
$p q-3$. Suppose on contrary that $m>(p q-3)$, then either $m=p q-2$ or $m=p q-1$. In both cases, the connected $T(p, q)$ at $1-2$ distance is not possible, which completes the proof.

\section{Conflicts of Interest}

The authors declare that they have no conflicts of interest.

\section{References}

[1] D. Cvetkovic, M. Doob, and H. Sachs, Spectra of Graphs, Johann Ambrosius Barth, Heidelberg, Germany, third edition, 1995.

[2] Y.-Z. Fan, F.-F. Zhang, and Y. Wang, "The least eigenvalue of the complements of trees," Linear Algebra and its Applications, vol. 435, no. 9, pp. 2150-2155, 2011.

[3] M. Javaid, "Minimizing graph of the connected graphs whose complements are bicyclic with two cycles," Turkish Journal of Mathematics, vol. 41, pp. 1433-1445, 2017.

[4] M. Javaid, "Characterization of the minimizing graph of the connected graphs whose complements are bicyclic," Mathematics, vol. 5, pp. 1-12, 2017.

[5] M. Javaid, "On the second minimizing graph in the set of complements of trees," AKCE International Journal of Graphs and Combinatorics, vol. 16, no. 3, pp. 258-264, 2019.

[6] L. Gul, G. Ali, U. Waheed, and S. Nasir, "The Least eigenvalue of the complement of the square power graph of G," Complexity, vol. 2021, Article ID 5885834, 4 pages, 2021.

[7] F. K. Bell, D. Cvetković, P. Rowlinson, and S. K. Simić, "Graphs for which the least eigenvalue is minimal, I," Linear Algebra and its Applications, vol. 429, no. 1, pp. 234-241, 2008.

[8] G. Constantine, "Lower bounds on the spectra of symmetric matrices with nonnegative entries," Linear Algebra and its Applications, vol. 65, pp. 171-178, 1985.

[9] Y. Z. Fan, Y. Wang, and Y.-B. Gao, "Minimizing the least eigenvalues of unicyclic graphs with application to spectral spread," Linear Algebra and its Applications, vol. 429, no. 2-3, pp. 577-588, 2008.

[10] R. Liu, M. Zhai, and J. Shu, "The least eigenvalues of cyclic graphs with $n$ vertices and $k$ pendant vertices," Linear Algebra and its Applications, vol. 431, no. 4-7, pp. 657-665, 2009.

[11] M. Petrovic, B. Borovicanin, and T. Aleksic, "Bicyclic graphs for which the least eigenvalue is minimum," Linear Algebra and its Applications, vol. 430, no. 4, pp. 1328-1335, 2009.

[12] F. K. Bell, D. Cvetković, P. Rowlinson, and S. K. Simić, "Graphs for which the least eigenvalue is minimal, II," Linear Algebra and its Applications, vol. 429, no. 8-9, pp. 2168-2179, 2008. 